\title{
The structural characteristics of photoageing in mice caused by the effects of ultraviolet $A$ radiation
}

\author{
S. Savic ${ }^{1}$, S. Smiljic ${ }^{2}$, S. Lestarevic ${ }^{1}$, A. Ilic 3 , M. Mijovic ${ }^{4}$, P. Mandic 5 , B. Djerkovic ${ }^{4}$ \\ ${ }^{1}$ Department of Histology and Embryology, Medical Faculty Pristina, Kosovska Mitrovica, Serbia \\ ${ }^{2}$ Department of Physiology, Medical Faculty Pristina, Kosovska Mitrovica, Serbia \\ ${ }^{3}$ Department of Medical Statistic and Informatics, Medical Faculty Pristina, Kosovska Mitrovica, Serbia \\ ${ }^{4}$ Department of Pathology, Medical Faculty Pristina, Kosovska Mitrovica, Serbia \\ ${ }^{5}$ Department of Anatomy, Medical Faculty Pristina, Kosovska Mitrovica, Serbia
}

[Received: 2 September 2019; Accepted: 22 October 2019]

Background: Due to its deep penetration into the dermis, ultraviolet A (UVA) radiation is considered a primary factor in skin photoageing. The aim of this study is to use a qualitative and quantitative analysis to determine the structural parameters of skin photoageing in mice exposed to UVA radiation, with or without the application of a photoprotective cream.

Materials and methods: The experiment consisted of the radiation of female $B A L B C$ mice in a solarium by UVA rays, up to total dosages of $7800 \mathrm{~J} / \mathrm{cm}^{2}$ and $12500 \mathrm{~J} / \mathrm{cm}^{2}$. A total of 78 animals were divided into four experimental and two control groups. All animals were shaved and the animals in two experimental groups were treated with a photoprotective cream half an hour before exposure. The samples of the treated skin were stained with haematoxylin-eosin and Van-Gieson staining methods. All measurements, except for the presence of dyskeratosis, were taken using ImageJ 150i software.

Results: In the study, the signs of skin photoageing were more evident in untreated groups of animals. Dyskeratosis was more frequent in both of the untreated groups of animals ( $p=0.004$ and $p=0.003$ ). The lowest values of epidermal thickness $(13.8 \pm 2.6 \mu \mathrm{m}$ and $12.7 \pm 2.3 \mu \mathrm{m})$ were present in both of the untreated groups of animals ( $p<0.001$ and $p<0.001)$. The highest values of stratum corneum thickness (34.3 $\pm 8.5 \mu \mathrm{m})$ were observed in the untreated, shorter radiated group of animals $(p<0.001)$ which was irradiated for the shortest period of time. Beside the control groups, the highest length of dermo-epidermal junction was recorded in the group of treated, longer radiated animals (1467.6 $\pm 94.6 \mu \mathrm{m}$; $p=0.373)$. The lowest values of dermal thickness (115.9 $\pm 10.5 \mu \mathrm{m}$ and $134.8 \pm 21.8 \mu \mathrm{m})$ and volumetric density of the collagen fibres (31.92 $\pm 3.19 \%$ and $29.40 \pm 4.54 \%)$ were present in both untreated groups of animals $(p<0.001$, $p<0.001, p=0.035$ )

Conclusions: Skin photoageing was most pronounced in the groups of animals irradiated without the application of photoprotective cream. (Folia Morphol 2020; 79, 3: 548-556)

Key words: photoageing, photoprotection, irradiation, ImageJ, mice 


\section{INTRODUCTION}

Photoageing is a cumulative process which depends on the degree of ultraviolet A (UVA) radiation or artificial emitters and skin type. It affects lighter skinned individuals more severely. The solar ultraviolet spectrum that penetrates the Earth's surface consist of $1-5 \%$ of ultraviolet B (UVB; $290-320 \mathrm{~nm}$ ) and $95-99 \%$ of ultraviolet A (UVA; $320-400 \mathrm{~nm}$ ) radiation $[2,14]$. Since most UVB rays are absorbed on the surface of epidermis only $10 \%$ of rays reach papillary dermis. UVA radiation penetrates deeper into the dermis (20-30\%) and is therefore considered a primary factor in skin photoageing $[3,4]$.

Photoageing mechanism through UVA radiation is based on the generation of reactive oxygen species (ROS) which induces oxidative damage to lipids, proteins, and the nucleic and mitochondrial DNA $[5,18,22,35,43]$. ROS-induced activation of proteases, matrix metalloproteinases (MMP) kinase pathways and elastase, originated mainly from keratinocytes [36] and fibroblasts [7]. They degrade collagen and other extracellular matrix proteins and can be seen as a key to pathological substrate photoageing and lead to an increased formation of deep skin folds, wrinkles and a loss of turgor [7, 27, 36]. Photoaged epidermis is characterised by cellular atypia, thickness variability, dyskeratosis, hyperkeratosis, irregular arrangement of pigment granules $[7,18,23]$, the changes in structure and appearance of dermo-epidermal junction (DEJ) [1] while damaged dermal and disorganised collagen fibrils and a massive accumulation of aberrant elastic material appear at the dermis level [4, 24, 25, 34, 38].

Skin photoprotection is one of the golden standards primarily for the prevention of ROS-induced damage. The application of topical antioxidant sources, especially photoprotective agents containing UVB and UVA filters, effectively protects the skin from sunburn, photoageing, DNA mutations and carcinogenesis $[2,7,8,27,37,40]$. In the present study, through the analysis and morphometric techniques of measuring of the structural parts of the skin of mice a clear qualitative and quantitative difference is intended to be shown in appearance and structure of unprotected and photo protected mouse skin exposed to high dosages of UVA radiation.

\section{MATERIALS AND METHODS}

Experimental animals. In this experiment female BALB/CAnNCr mice, 6-8 weeks old, were used and purchased from the farm for breeding and keeping laboratory animals (Military Medical Academy, Belgrade, Serbia). The conducted experimental proceedings were approved by the Ethics Committee of the Medical Faculty, University of Pristina, based in Kosovska Mitrovica (no. 90/14.07.09). While conducting the experiment the animals were treated in accordance with the U.K. Animals (Scientific Procedures) Act, 1986 and associated guidelines, EU Directive 2010/63/EU for animal experiments, or the National Institutes of Health guide for the care and use of Laboratory animals (NIH Publications No. 8023, revised 1978).

Experimental proceedings. During the experiment, the mice were exposed to UV radiation lamps (Independence XRR40W, New-Technology, Meppen, Germany). Since these lamps emit $98.9 \%$ UVA rays and $1.1 \%$ UVB rays, the effect of UVB radiation was eliminated by a $3 \mathrm{~mm}$ UVB absorbing window glass [13]. The animals were divided into four experimental and two control groups. The experimental groups of animals were exposed to radiation for 2 hours a day (the dosage of $156 \mathrm{~J} / \mathrm{cm}^{2}$ ) 5 consecutive days per week, over a period of 10 weeks (radiated at $7800 \mathrm{~J} / \mathrm{cm}^{2}$ ) and 16 weeks (radiated at $12500 \mathrm{~J} / \mathrm{cm}^{2}$ ). The distance between the lamps and the mice was approximately $30 \mathrm{~cm}$, and fans increased the air circulation. The literature data indicates that high cumulative UVA radiation doses are needed to cause the skin photoageing signs, especially on collagen fibres $\left(>8000 \mathrm{~J} / \mathrm{cm}^{2}\right.$ ) [25].

Prior to irradiation, animals of the experimental and control groups were shaved three times a week for the removal of hair from the skin on their backs, while $30 \mathrm{~min}$ before exposure two experimental groups of animals, irradiated at different radiation doses, had their skin treated by a photoprotective cream with sun protection factor (SPF) $50+$ and UVA filter. Sunscreen was made with the following sunscreen actives: Avobenzone (3\%), Homosalate (10\%), Octisalate (5\%), Octocrylene (5\%), Oxybenzone (6\%). The total amount of the treatment for every application was two drops (approximately $0.1 \mathrm{~mL}$ ).

\section{MATERIALS AND METHODS}

The samples of the skin from the back of the mice were fixed in $10 \%$ buffered formalin, dehydrated in multiple baths of increasing concentrations of ethanol, enlightened in xylene and stored in paraffin-embedded blocks. The paraffin blocks were cut on a rotary microtome into serial $5 \mu \mathrm{m}$ sections which were then stained with haematoxylin-eosin (HE) and Van Gieson staining methods. Five representative 
samples stained with HE method were used to obtain a general overview of the histological structure of the skin, as well as for determining the thickness of corneal layer, viable epidermis, dermis, and the length of the basal membrane, while for the visualisation and analysis of collagen fibres Van Gieson staining method was used.

The samples were analysed on a Leica microscope and photographed by Leica MC190 HD digital microscope camera at $10 \times, 40 \times$ and $100 \times$ magnifications. All measurements were taken by using ImageJ 150i, which are available to any users of photo-processing (http://rskwebnih.gov/ij//) except for the presence of dyskeratotic cells, which were assessed in 100 consecutive fields of vision, at $40 \times$ magnification, per sample. Epidermal thickness, the thickness of corneal layer and dermal thickness were determined by taking measurements of five different places of each section. Epidermal thickness is defined as the minimal distance between the stratum corneum and basal layer, whereas dermal thickness is defined as the minimal distance between the basement membrane and the most noticeable layer of subcutaneous fat tissue. DEJ length was calculated by using calibrated photographs, $1217.4 \mu \mathrm{m}$ in length (the size of all digital images at $10 \times$ magnification was $1024 \times 768$ pixels, or $1217.4 \times 913 \mu \mathrm{m}$ ), on five consecutive photos for each sample. All morphometric measurements were taken according to existing recommendations, The User Guide for ImageJ is available at: http://imagej. nih.gov/ij/docs/index.html.

\section{Statistical analysis}

Descriptive statistical methods and methods for testing statistical hypotheses were used for the analysis of primary data. Among the descriptive statistical methods used were: measures of central tendency (mean value and median), measures of variability (standard deviation), and sample maximum (max) and sample minimum (min). Each variable was tested by Shapiro-Wilk test for assessing the normality of data distribution and Levene's test for the assessment of homogeneity of variance. Statistical hypotheses were tested by $\chi^{2}$ and Fisher's exact test. Student's t test was used to test the hypothesis about the statistical significance of mean values' numerical differences, as well as the single-factor variance analysis (ANOVA) and the Post Hoc Test (Tukey HSD).

Statistical hypotheses were tested at the level of statistical significance (alpha level) of 0.05 . For the pur-
Table 1. The distribution of animals per group $(n=78)$

\begin{tabular}{ll}
\hline Group of animals & $\mathbf{N}$ \\
\hline $\mathrm{U}_{7800}$ - irradiated at $7800 \mathrm{~J} / \mathrm{cm}^{2}$, untreated by the cream & 12 \\
$\mathrm{~T}_{7800}$ - irradiated at $7800 \mathrm{~J} / \mathrm{cm}^{2}$, treated by the cream & 12 \\
$\mathrm{U}_{12500}$ - irradiated at $12500 \mathrm{~J} / \mathrm{cm}^{2}$, untreated by the cream & 12 \\
$\mathrm{~T}_{12500}$ - irradiated at $12500 \mathrm{~J} / \mathrm{cm}^{2}$, treated by the cream & 15 \\
$\mathrm{~K}_{7800}$ - control group (for groups $\mathrm{U}_{7800}$ and $\mathrm{T}_{7800}$ ), shaved & 13 \\
$\mathrm{~K}_{12500}$ - control group (for groups $\mathrm{U}_{12500}$ and $\mathrm{T}_{12500}$ ), shaved & 14 \\
Total & 78 \\
\hline
\end{tabular}

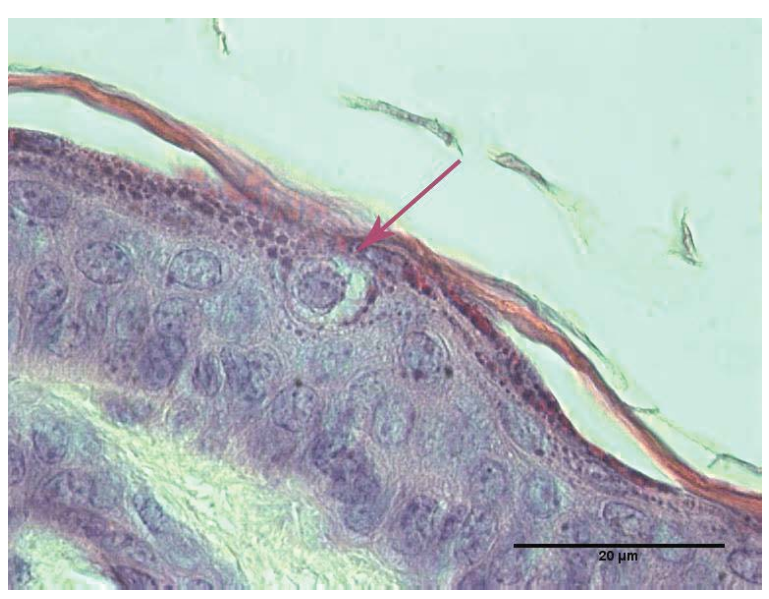

Figure 1. The presence of dyskeratosis (arrows); haematoxylin-eosin-stained sections of histological mouse skin; $100 \times$ /immersion; Group $\mathrm{U}_{7800}$ - untreated by a photoprotective cream, radiated at $7800 \mathrm{~J} / \mathrm{cm}^{2}$.

pose of statistical data analysis, SPSS Statistics 22 (SPSS Inc., Chicago, IL, USA) software package was used.

\section{RESULTS}

Experimental research was conducted on $78 \mathrm{fe}$ male BALB/CAnNCr mice which were separated into four experimental and two control groups depending on the way they were treated and the input doses of radiation, shown in Table 1.

Through analysis of HE-stained tissue samples the presence of dyskeratotic cells in the epidermis of the skin of experimental group of animals was confirmed, shown in Figure 1. The presence of dyskeratosis is significantly more frequent in Group $U_{7800}$ compared to Group $T_{7800}(p=0.004)$, as well as in Group $U_{12500}$ compared to Group $T_{12500}$ of animals ( $p=0.003$ ), shown in Table 2.

Through analysis of HE-stained tissue samples significant differences in skin appearance and structure of the treated animals were noticed. Normal appearance and staining of mouse skin were seen in 
Table 2. The presence of dyskeratosis in experimental groups of animals

\begin{tabular}{lcc}
\hline Group of animals & The presence of dyskeratoses $-\mathbf{N}(\%)$ & $\mathbf{P}$ \\
\hline $\mathrm{U}_{7800}$ & $10(76.9 \%)$ & $0.004^{*}$ \\
$\mathrm{~T}_{7800}$ & $3(23.1 \%)$ & \\
$\mathrm{U}_{12500}$ & $10(71.4 \%)$ & $0.003^{*}$ \\
$\mathrm{~T}_{12500}$ & $4(28.6 \%)$ & \\
$\mathrm{U}_{7800}$ & $10(50.0 \%)$ & 1.0 \\
$\mathrm{U}_{12500}$ & $10(50.0 \%)$ & \\
$\mathrm{T}_{7800}$ & $3(42.9 \%)$ & 0.922 \\
$\mathrm{~T}_{12500}$ & $4(57.1 \%)$ & \\
\hline
\end{tabular}

${ }^{*}$ Statistically significant differences the control group of animals. The biggest degree in pathohistological characteristics of changes was seen in Group $\mathrm{U}_{7800}$ while in Group $\mathrm{T}_{12500}$ the changes were mildly registered, shown in Figure 2 .

By the analysis of Van Gieson stained tissue samples significant differences in appearance, arrangement and colouring of collagen fibres of dermis were recognised. Normal appearance and staining of collagen fibres were registered in the control group. The biggest degree in pathohistological characteristics of changes was seen in Group $U_{12500}$ while in Group $T_{12500}$ the changes were mildly registered, shown in Figure 3 . By quantifying all of the analysed structural parameters
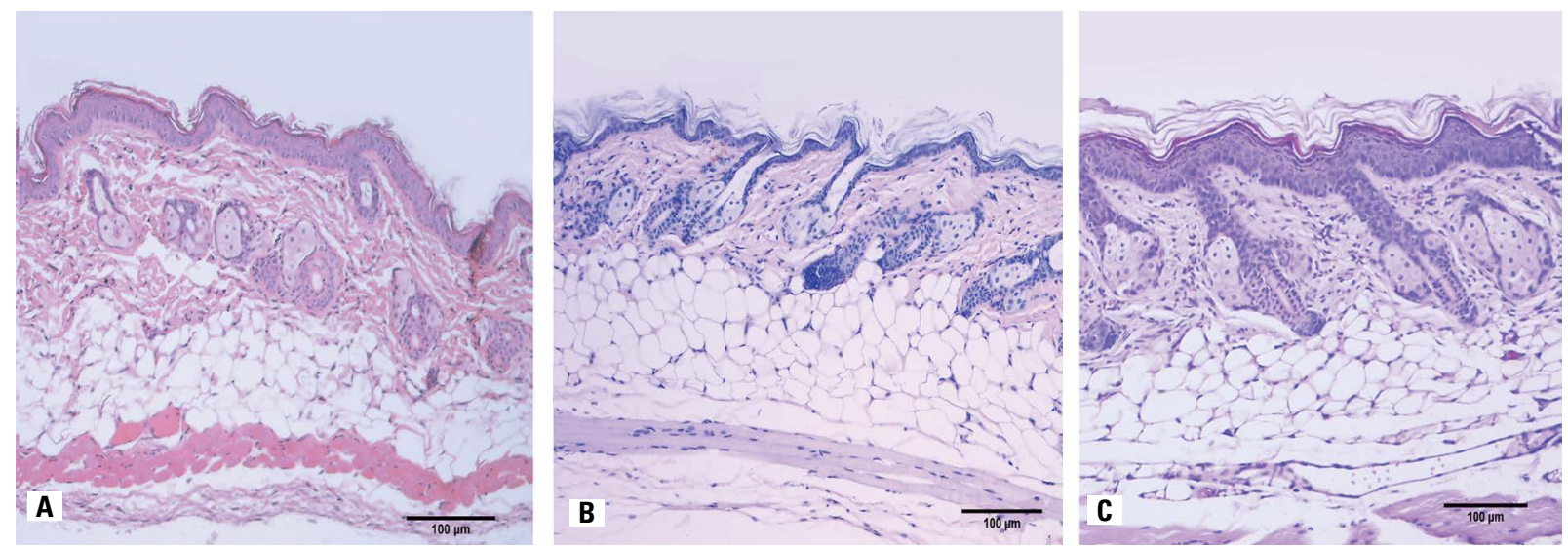

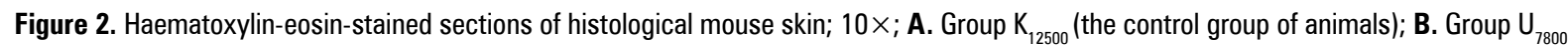
(untreated with the photoprotective cream, radiated at $7800 \mathrm{~J} / \mathrm{cm}^{2}$ ); C. Group $\mathrm{T}_{12500}$ (treated with the photoprotective cream, radiated at $12500 \mathrm{~J} / \mathrm{cm}^{2}$ ); A. A normal appearance and staining of mouse skin; B. A pronounced epithelial atrophy (3-4 rows of cells), hypogranulosis, the granulosa layer is focally absent, diffuse orthokeratotic hyperkeratosis, flattening epidermal ridges. In the upper layers of the dermis, densely packed collagen fibres, moderately basophilic; C. Preserved epidermal thickness and architectonics, hypogranulosis, orthokeratotic hyperkeratosis with occasional foci of parakeratosis, a wavy basement membrane. Particularly densely packed collagen fibres, especially in the upper layers of the dermis.
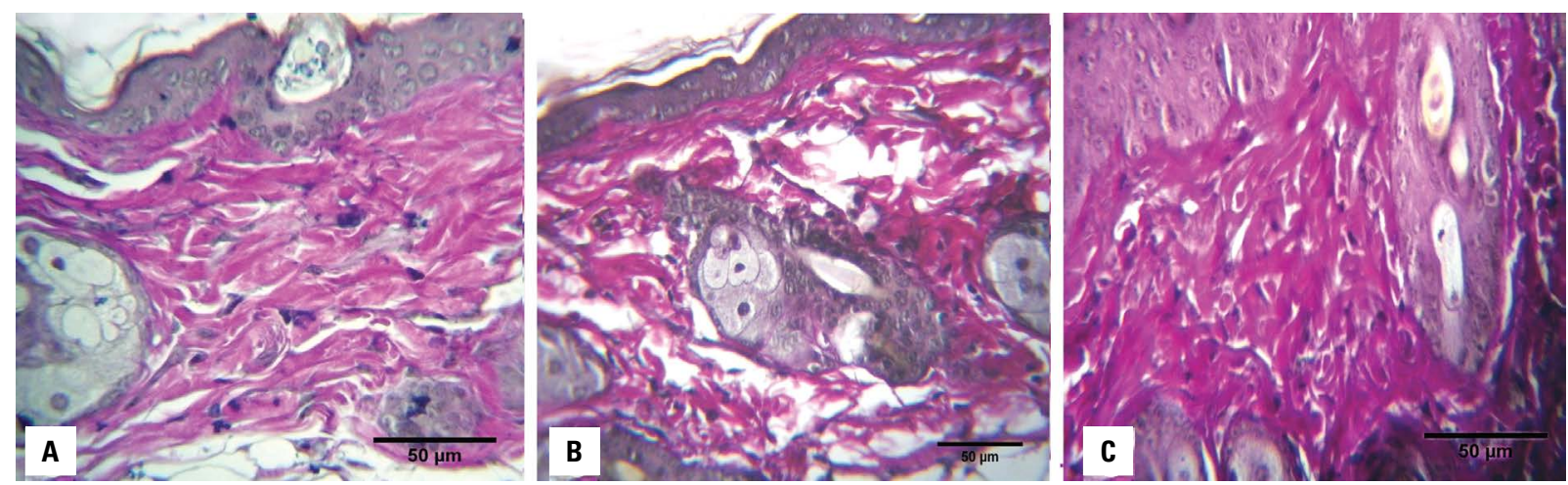

Figure 3. Van Gieson-stained histological sections of mouse skin; $40 \times$; A. Group $\mathrm{K}_{12500}$ (the control group of animals); B. Group $\mathrm{U}_{12500}$ (untreated with the photoprotective cream, radiated at $12500 \mathrm{~J} / \mathrm{cm}^{2}$ ); C. Group $\mathrm{T}_{12500}$ (treated with the photoprotective cream, radiated at $12500 \mathrm{~J} / \mathrm{cm}^{2}$ ); A. A normal appearance and staining of collagen fibres; B. Destruction and change of dermal architectonics, disorganised collagen fibres, increased extracellular matrix; C. Disorganised, densely packed collagen fibres throughout the dermis. 
Table 3. The values of the parameters tested between the experimental groups of animals (untreated and treated, irradiated at $7800 \mathrm{~J} / \mathrm{cm}^{2}$ ) and their control groups

\begin{tabular}{|c|c|c|c|c|c|c|}
\hline \multirow[t]{2}{*}{ Parameter } & \multicolumn{3}{|c|}{ Group of animals - mean value \pm SD } & \multicolumn{3}{|c|}{$\mathbf{P}$} \\
\hline & $\mathbf{U}_{7800}$ & $T_{7800}$ & $K_{7800}$ & $\mathrm{U}_{7800}-\mathrm{T}_{7800}$ & $U_{7800}-K_{7800}$ & $T_{7800}-K_{7800}$ \\
\hline Epidermal thickness $[\mu \mathrm{m}]$ & $13.8 \pm 2.6$ & $21.6 \pm 5.2$ & $21.2 \pm 4.0$ & $<0.001^{*}$ & $<0.001^{*}$ & 0.972 \\
\hline Stratum corneum thickness $[\mu \mathrm{m}]$ & $34.3 \pm 8.5$ & $17.5 \pm 5.6$ & $23.7 \pm 3.8$ & $<0.001^{*}$ & $<0.001^{*}$ & $0.037^{*}$ \\
\hline DEJ length $[\mu \mathrm{m}]$ & $1434.2 \pm 146.3$ & $1409.1 \pm 121.0$ & $1570.8 \pm 109.5$ & 0.868 & $0.027^{*}$ & $0.005^{*}$ \\
\hline Dermal thickness $[\mu \mathrm{m}]$ & $115.9 \pm 10.5$ & $149.9 \pm 28.4$ & $186.0 \pm 13.9$ & $<0.001^{*}$ & $<0.001^{*}$ & $<0.001^{*}$ \\
\hline Collagen [\%] & $31.9 \pm 3.2$ & $35.0 \pm 3.1$ & $38.4 \pm 2.8$ & $0.035^{*}$ & $<0.001^{*}$ & $0.017^{*}$ \\
\hline
\end{tabular}

DEJ — dermo-epidermal junction; SD — standard deviation, ${ }^{*}$ statistically significant differences

Table 4. The values of the parameters tested between the experimental groups of animals (untreated and treated, irradiated at $12500 \mathrm{~J} / \mathrm{cm}^{2}$ ) and their control groups

\begin{tabular}{|c|c|c|c|c|c|c|}
\hline \multirow[t]{2}{*}{ Parameter } & \multicolumn{3}{|c|}{ Groups of animals - mean value \pm SD } & \multicolumn{3}{|c|}{$\mathbf{P}$} \\
\hline & $\mathbf{U}_{12500}$ & $T_{12500}$ & $K_{12500}$ & $U_{12500}-T_{12500}$ & $U_{12500}-K_{12500}$ & $T_{12500}-K_{12500}$ \\
\hline Epidermal thickness $(\mu \mathrm{m})$ & $12.7 \pm 2.3$ & $32.2 \pm 8.1$ & $27.6 \pm 4.5$ & $<0.001^{*}$ & $<0.001^{*}$ & 0.094 \\
\hline Stratum corneum thickness $(\mu \mathrm{m})$ & $33.2 \pm 8.9$ & $26.7 \pm 6.9$ & $18.4 \pm 5.0$ & 0.059 & $<0.001^{*}$ & $0.009^{*}$ \\
\hline DEJ length $(\mu \mathrm{m})$ & $1405.6 \pm 75.8$ & $1467.6 \pm 94.6$ & $1517.3 \pm 114.1$ & 0.234 & $0.017^{*}$ & 0.373 \\
\hline Dermal thickness $(\mu \mathrm{m})$ & $134.8 \pm 21.8$ & $192.0 \pm 29.4$ & $201.5 \pm 10.6$ & $<0.001^{*}$ & $<0.001^{*}$ & 0.508 \\
\hline Collagen (\%) & $29.4 \pm 4.5$ & $40.9 \pm 2.5$ & $43.5 \pm 3.3$ & $<0.001^{*}$ & $<0.001^{*}$ & 0.146 \\
\hline
\end{tabular}

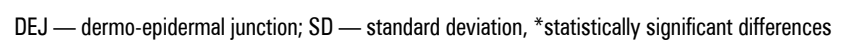

- the measurements of the epithelial thickness, the stratum corneum thickness, the length of DEJ, dermis thickness and volumetric density of collagen fibres of mouse skin the existence of statistically significant difference was registered between the examined groups.

The epithelial thickness was significantly higher in Group $T_{7800}$ compared to Group $U_{7800}(p<0.001)$ as well as in Group $\mathrm{K}_{7800}$ compared to Group $\mathrm{U}_{7800}$ of animals ( $p<0.001)$, shown in Table 3 . In addition, the epithelial thickness was significantly higher in Group $\mathrm{T}_{12500}$ compared to Group $\mathrm{U}_{12500}(\mathrm{p}<0.001)$ as well as in Group $K_{12500}$ compared to Group $U_{12500}$ ( $p<0.001$ ), shown in Table 4. The stratum corneum thickness was significantly higher in Group $U_{7800}$ compared to Group $T_{7800}(p<0.001)$, in Group $U_{7800}$ compared to Group $K_{7800}(p<0.001)$ as well as in Group $K_{7800}$ compared to Group $T_{7800}$ of animals ( $p=0.037$ ), shown in Table 3. Furthermore, the stratum corneum thickness was significantly higher in Group $U_{12500}$ compared to Group $K_{12500}(p<0.001)$ and in Group $T_{12500}$ compared to Group $K_{12500}(p=0.009)$, shown in Table 4. The length of DEJ was significantly

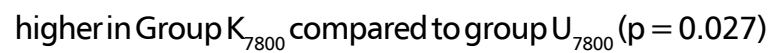
as well as in Group $K_{7800}$ compared to Group $\mathrm{T}_{7800}$ $(p=0.005)$, shown in Table 3. Moreover, the length of
DEJ was significantly higher in Group $K_{12500}$ compared to Group $U_{12500}(p=0.017)$.

The dermal thickness was significantly higher in Group $T_{7800}$ compared to Group $U_{7800}(p<0.001)$, in Group $K_{7800}$ compared to Group $U_{7800}(p<0.001)$ as well as in Group $K_{7800}$ compared to Group $\mathrm{T}_{7800}$ of animals ( $p<0.001)$, shown in Table 3. The dermal thickness was significantly higher in Group $\mathrm{T}_{12500}$ compared to Group $U_{12500}(p<0.001)$, as well as in Group $K_{12500}$ compared to Group $U_{12500}(p<0.001)$, shown in Table 4. Similar results were also registered by measuring the volumetric density of collagen fibres. The density was significantly higher in Group $T_{7800}$ compared to Group $U_{7800}(p=0.035)$, in Group $K_{7800}$ compared to Group $\mathrm{U}_{7800}(\mathrm{p}<0.001)$, as well as in Group $K_{7800}$ compared to Group $\mathrm{T}_{7800}$ of animals $(p=0.017)$, shown in Table 3 . Additionally, the density was registered as significantly higher in Group $\mathrm{T}_{12500}$ compared to Group $U_{12500}$ ( $p<0.001$ ) as well as in Group $K_{12500}$ compared to Group $U_{12500}$ $(p<0.001)$, shown in Table 4.

\section{DISCUSSION}

Skin is the largest organ in the human body and its health is dependent on a variety of factors, including 
exposure to UV light. Erythema is the most visible phenomenon caused by the exposure to UV radiation. UVA induced erythema contributes to at least $15 \%$ of total sun-induced erythema. UVA radiation is 1000 -fold less effective than UVB in causing skin erythema [4]. Sunburn cells in the epidermis appear shortly after the induction of erythema caused by direct DNA damage that leads to the formation of cyclobutane pyrimidine dimers (CPDs), and pyrimidine (6-4) pyrimidone (6-4PP), especially due to the effects of UVB irradiation [28]. UVA toxicity mainly depends on indirect mechanisms which generate oxidative damage in the DNA, oxidative DNA lesions, mainly 8-oxo-7,8-dihydro-20-deoxyguanosine [5]. However, some studies have confirmed that UVA radiation can initiate direct mutations of DNA and that CPDs represent the most relevant UVA-induced type of DNA lesion [28, 32]. Type of CPD at TT sites, were the most frequent type of mutations observed and the poor formation of 64PP $[5,12]$. Due to the depth of the penetration and accumulation of UVA rays on the entire skin, it is considered that these types of mutation are more harmful than those made by UVB radiation. Runger et al. [39] explain this by the less effective cell cycle arrest, weak p53 and p95 activation and less effective cell cycle control under the influence of UVA radiation which results in replication of damaged DNA, mutation accumulation and ultimately possible carcinogenesis.

The dyskeratotic cells were significantly more present in the untreated groups of animals, regardless of the applied radiation dose, which supports the fact that the radiation at $7800 \mathrm{~J} / \mathrm{cm}^{2}$ showed mutagenic potential. The final results are consistent with literature findings and confirm the potential of UVA radiation to induce direct mutations of DNA $[5,12$, $32,39]$. The key parameter in photoprotection is the prevention of DNA mutation that can inhibit or delay the onset of malignancy and photoageing of the skin $[3,17,32]$ which was also confirmed by the conducted study.

Skin is a major target organ of ROS induced by UVA irradiation. Keratinocyte and mitochondrial membranes, as well as the molecules of the nucleic and mitochondrial DNA are targeted by ROS [21, 33]. Keratinocytes are rapidly regenerated cells which viability was substantially reduced by ROS [29]. The impact of ROS on keratinocytes depends on the applied radiation dose where low ROS levels can be mutagenic, medium levels can result in replicative senescence, and high ROS levels usually lead to cell death by apoptosis, necroptosis and necrosis $[17,19]$. In physiological conditions, cell apoptosis represents a balancing mechanism between cell proliferation rate and epithelial thickness. ROS-induced apoptosis includes mitochondrial involvement in the activation and amplification of caspase cascade [21]. Additional regulatory-homeopathic mechanism is activated in the oxidative damage stage phospholipids in keratinocytes-autophagy, which helps the damaged keratinocytes, causes of potential carcinoma, to be removed [15]. Massive oxidative damage to keratinocytes and the exhaustion of antioxidant protective mechanisms of cells induce the initiation of these protection mechanisms which, despite the high proliferative activity of the keratinocytes under the influence of UVA radiation and the mechanical stimulation of the skin $[6,16]$ cause atrophy of the epidermis. The lowest values of epidermal thickness were present in the untreated groups of animals, while the highest levels of epidermal thickness were present in the treated groups of animals without any difference compared to the control groups, which is explained by the usage of a photoprotective cream and influence of mechanical stimulation of the skin. By comparing the results of the study with the results of other researchers, it was realised that there is no conformity of views on the dynamics of epidermal thickness variation and photoageing. Some researchers claim that one of the characteristics of skin photoageing is epidermal atrophy $[23,30]$. Chen et al. [10] indicate flattening of the basal layer of the cells and thinning of the spinous layer with the absence of granular layer of epidermis in photoaged skin. Contrary to this study, other researchers state that epidermal thickening occurs as a result of irradiation [20,37]. One of the factors that cannot be ignored when comparing the results of this study to those of the other studies is that significantly lower radiation doses were applied in them.

Hyperkeratosis is a protective mechanism of the skin which increases the epidermal thickness and reduces the amount of UV radiation that penetrates the deeper skin structures [41]. It occurs mainly due to the effects of UVB radiation. The highest values of stratum corneum thickness were observed in both of untreated groups of animals, as well as in the treated, longer irradiated group of animals. Results similar to the results of this study could partly be found with other researchers [20] who, along with 
the stratum corneum thickening, also claimed the existence of an increased thickness of granular cell layer after irradiation. The results of this study show a preserved granular layer or hypergranulosis in the treated groups of animals, while in the untreated groups of animals the presence of a thin and often discontinuous granular cell layer was noted, similar to Chen et al. [10] along with hyperkeratosis. Bearing in mind the classic image of atrophic epidermis, which includes all "live" cell layers, it is believed that the granular layer was subject to the same changes due to the high radiation doses applied. The application of the photoprotective cream did not require "additional" compensatory mechanisms of the epidermis in the group irradiated at $7800 \mathrm{~J} / \mathrm{cm}^{2}$, which did not develop a significant hyperkeratosis.

The basement membrane represents the border between the epidermis and dermis, which influences epidermal differentiation and proliferative activity of the basal layer of cells. Keratinocytes of the photodamaged skin produce MMP, urinary plasminogen activator/plasmin and heparinase which, in addition to decomposing dermal collagen and elastic fibres, also damage components of basal membrane [1]. Its flattening is a sign of ageing of the skin [26]. The results of the morphometric study show that DEJ length of the skin, without the loss of epidermal rete ridges, had the highest values in the control groups of animals, as well as in treated, longer irradiated groups of animals, without any difference compared to the control group. The literature shows similar results to the results of this study $[9,42]$. A number of studies also mention the changes in its structural components; in the photoaged skin the number of anchoring fibrils is significantly lower while the link between the epidermis and the dermis weakens, becoming one of the causes of wrinkle appearance [1]. The mechanical stimulation of mouse skin induces the strengthening of the DEJ, while the production of collagen VII, fibrillin, decorin, tropoelastin, fibronectin and procollagen 1 is increased [9] so that longer mechanical stimulus exerted another positive effect on mouse skin which, along with the application of photoprotective cream, led to the absence of changes in the treated, longer irradiated group of animals.

The basis of histological substrate photoageing is a change of dermal architectonics due to a greater sensitivity of dermal components to the effects of UVA radiation [3, 31]. Skin photoageing is characterised by reduced fibroblasts viability $[11,27]$ and the loss of mature collagen, its basophilic degeneration, splicing and interconnection of fibres and the changes to the qualitative relationship between fibres in favour of type III collagen. ROS affect collagen fibres in two ways: they cause collagen degradation and a reduced level of type I collagen, the major component of the dermis as well as the inhibition of procollagen biosynthesis, which progressively worsens skin photoageing $[8,36]$. UVA radiation induces a series of MMPs and activation of MMP-1 production has directly been involved in the degradation of type I collagen, while MMP-2 i MMP-9 are responsible for decomposition of collagen type I and IV. The lowest values of dermal thickness and volumetric density of the collagen fibres were observed in the untreated group of animals, regardless of the applied radiation dose. Disorganised and diluted collagen fibres, an increase in the amount of extracellular matrix and a moderate basophilia (HE) were noted. The highest values of dermal thickness and volumetric density of the collagen fibres were found in the control groups, as well as in the treated, longer irradiated group of animals. The results of this study are in accordance with the results of other researchers $[4,20,24,25$, $30,37,42]$. The degree of collagen damage increases with the increased cumulative doses of UV radiation, while photoprotective agents exhibit a significant effect in the prevention of oxidative damage to the skin, which was particularly confirmed in the treated, longer irradiated group of animals $[3,8,18,40]$. Note that, although there were no differences in the density of collagen fibres compared to its control group, there were distinct changes in the quality of disorganised and densely packed collagen fibres. Certainly, it should also be considered that the mechanical stimulation of mouse skin stimulates the growth of fibroblasts and induces collagen production [9]. The limit of the conducted study is the fact that it was based on classical histological qualitative and quantitative analyses. Experimental studies based on the cell culture, Western blot and real-time polymerase chain reaction analysis, would give a support to the results of this study, and give better insight into the mechanisms and the consequences of skin photoageing.

\section{CONCLUSIONS}

In summary, histological assessment is the application of the photoprotective cream led to the effective reduction and prevention of changes related to photoageing. The occurrence of dyskeratotic cells, epithe- 
lial atrophy, the shortening of DEJ length with the loss of epidermal rungs, decrease of the dermal thickness and volumetric density of collagen fibres of the mouse skin were present in untreated groups of animals. The highest values of stratum corneum thickness were observed in the untreated, shorter radiated group of animals. In the treated, longer irradiated group, all the measured parameters, except for stratum corneum thickness, corresponded to the parameters of its control group, which once again confirms the necessity and the justification for the application of photoprotective agents. Recommendation for healthy sunbathing habits implementation, prevention of tanning beds usage as means for acquiring darker $\tan$ and regular usage of photoprotective creams all contribute to prevention of skin-photoageing which is a "fertile ground" for precancerosis and nonmelanoma and melanoma skin cancer occurrence.

\section{REFERENCES}

1. Amano S. Characterization and mechanisms of photoageing-related changes in skin. Damages of basement membrane and dermal structures. Exp Dermatol. 2016; 25 Suppl 3: 14-19, doi: 10.1111/exd.13085, indexed in Pubmed: 27539897.

2. Amaro-Ortiz A, Yan B, D'Orazio JA. Ultraviolet radiation, aging and the skin: prevention of damage by topical cAMP manipulation. Molecules. 2014; 19(5): 6202-6219, doi: 10.3390/molecules19056202, indexed in Pubmed: 24838074.

3. Battie C, Jitsukawa S, Bernerd F, et al. New insights in photoaging, UVA induced damage and skin types. Exp Dermatol. 2014; 23 (Suppl 1): 7-12, doi: 10.1111/exd.12388, indexed in Pubmed: 25234829.

4. Battie C, Verschoore M. Cutaneous solar ultraviolet exposure and clinical aspects of photodamage. Indian J Dermatol Venereol Leprol. 2012; 78 (Suppl 1): S9-SS14, doi: 10.4103/0378-6323.97350, indexed in Pubmed: 22710112.

5. Beani JC. [Ultraviolet A-induced DNA damage: role in skin cancer]. Bull Acad Natl Med. 2014; 198(2): 273-295, indexed in Pubmed: 26263704.

6. Bertsch S, Csontos K, Schweizer J, et al. Effect of mechanical stimulation on cell proliferation in mouse epidermis and on growth regulation by endogenous factors (chalones). Cell Tissue Kinet. 1976; 9(5): 445-457, doi: 10.1111/j.1365-2184.1976.tb01295.x, indexed in Pubmed: 135644.

7. Bilaç $C$, Şahin MT, Öztürkcan S. Chronic actinic damage of facial skin. Clin Dermatol. 2014; 32(6): 752-762, doi: 10.1016/j.clindermatol.2014.02.014, indexed in Pubmed: 25441468.

8. Bosch R, Philips N, Suárez-Pérez JA, et al. Mechanisms of photoaging and cutaneous photocarcinogenesis, and photoprotective strategies with phytochemicals. Antioxidants (Basel). 2015; 4(2): 248-268, doi: 10.3390/antiox4020248, indexed in Pubmed: 26783703.
9. Caberlotto E, Ruiz L, Miller Z, et al. Effects of a skin-massaging device on the ex-vivo expression of human dermis proteins and in-vivo facial wrinkles. PLoS One. 2017; 12(3): e0172624, doi: 10.1371/journal.pone.0172624, indexed in Pubmed: 28249037.

10. Chen CYO, Smith A, Liu Y, et al. Photoprotection by pistachio bioactives in a 3-dimensional human skin equivalent tissue model. Int J Food Sci Nutr. 2017; 68(6): 712-718, doi: 10.1080/09637486.2017.1282437, indexed in Pubmed: 28122479.

11. Chen J, Luo J, Tan Y, et al. Effects of low-dose ALA-PDT on fibroblast photoaging induced by UVA irradiation and the underlying mechanisms. Photodiagnosis Photodyn Ther. 2019; 27: 79-84, doi: 10.1016/j.pdpdt.2019.05.006, indexed in Pubmed: 31075320.

12. Cortat B, Garcia CC, Quinet A, et al. The relative roles of DNA damage induced by UVA irradiation in human cells. Photochem Photobiol Sci. 2013; 12(8): 1483-1495, doi: 10.1039/c3pp50023c, indexed in Pubmed: 23824260.

13. Duarte I, Rotter A, Malvestiti A, et al. The role of glass as a barrier against the transmission of ultraviolet radiation: an experimental study. Photodermatol Photoimmunol Photomed. 2009; 25(4): 181-184, doi: 10.1111/j.16000781.2009.00434.x, indexed in Pubmed: 19614895.

14. Dupont E, Gomez J, Bilodeau D. Beyond UV radiation: a skin under challenge. Int J Cosmet Sci. 2013; 35(3): 224-232, doi: 10.1111/ics.12036, indexed in Pubmed: 23406155.

15. Eckhart L, Tschachler E, Gruber F. Autophagic control of skin aging. Front Cell Dev Biol. 2019; 7: 143, doi: 10.3389/ fcell.2019.00143, indexed in Pubmed: 31417903.

16. Evans-Johnson JA, Garlick JA, Johnson EJ, et al. A pilot study of the photoprotective effect of almond phytochemicals in a 3D human skin equivalent. J Photochem Photobiol B. 2013; 126: 17-25, doi: 10.1016/j.jphotobiol.2013.07.006, indexed in Pubmed: 23892186.

17. Gomes-Neto A, Aguilera P, Prieto L, et al. Efficacy of a daily protective moisturizer with high UVB and UVA photoprotection in decreasing ultraviolet damage: evaluation by reflectance confocal microscopy. Acta Derm Venereol. 2017; 97(10): 1196-1201, doi: 10.2340/00015555-2736, indexed in Pubmed: 28661544.

18. Gonzaga ER. Role of UV light in photodamage, skin aging, and skin cancer: importance of photoprotection. Am J Clin Dermatol. 2009; 10 (Suppl 1): 19-24, doi: 10.2165/0128071200910001-00004, indexed in Pubmed: 19209950.

19. Harberts E, Fishelevich R, Liu J, et al. MyD88 mediates the decision to die by apoptosis or necroptosis after UV irradiation. Innate Immun. 2014; 20(5): 529-539, doi: 10.1177/1753425913501706, indexed in Pubmed: 24048771.

20. Hung CF, Fang CL, Al-Suwayeh SA, et al. Evaluation of drug and sunscreen permeation via skin irradiated with UVA and UVB: comparisons of normal skin and chronologically aged skin. J Dermatol Sci. 2012; 68(3): 135-148, doi: 10.1016/j. jdermsci.2012.09.005, indexed in Pubmed: 23026054.

21. Kammeyer A, Luiten RM. Oxidation events and skin aging. Ageing Res Rev. 2015; 21: 16-29, doi: 10.1016/j. arr.2015.01.001, indexed in Pubmed: 25653189.

22. Karran P, Brem R. Protein oxidation, UVA and human DNA repair. DNA Repair. 2016; 44: 178-185, doi: 10.1016/j. dnarep.2016.05.024. 
23. Khavkin J, Ellis DAF. Aging skin: histology, physiology, and pathology. Facial Plast Surg Clin North Am. 2011; 19(2): 229-234, doi: 10.1016/j.fsc.2011.04.003, indexed in Pubmed: 21763983.

24. Kligman LH, Gebre M, Alper R, et al. Collagen metabolism in ultraviolet irradiated hairless mouse skin and its correlation to histochemical observations. J Invest Dermatol. 1989; 93(2): 210-214, doi: 10.1111/1523-1747. ep12277573, indexed in Pubmed: 2474028.

25. Kligman L. The hairless mouse model for photoaging. Clin Dermatol. 1996; 14(2): 183-195, doi: 10.1016/0738081x(95)00154-8.

26. Lagarrigue SG, George J, Questel E, et al. In vivo quantification of epidermis pigmentation and dermis papilla density with reflectance confocal microscopy: variations with age and skin phototype. Exp Dermatol. 2012; 21(4): 281-286, doi: 10.1111/j.1600-0625.2012.01451.x, indexed in Pubmed: 22417304.

27. Lan CCE, Hung $Y T$, Fang $A H$, et al. Effects of irradiance on UVA-induced skin aging. J Dermatol Sci. 2019; 94(1): 220-228, doi: 10.1016/j.jdermsci.2019.03.005, indexed in Pubmed: 30956032.

28. Leccia MT, Lebbe C, Claudel JP, et al. New Vision in Photoprotection and Photorepair. Dermatol Ther (Heidelb). 2019; 9(1): 103-115, doi: 10.1007/s13555-019-0282-5, indexed in Pubmed: 30674003.

29. Liu $Y$, Chan F, Sun $H$, et al. Resveratrol protects human keratinocytes $\mathrm{HaCaT}$ cells from UVA-induced oxidative stress damage by downregulating Keap1 expression. Eur J Pharmacol. 2011; 650(1): 130-137, doi: 10.1016/j. ejphar.2010.10.009, indexed in Pubmed: 20951123.

30. Longo C, Casari A, Beretti F, et al. Skin aging: in vivo microscopic assessment of epidermal and dermal changes by means of confocal microscopy. J Am Acad Dermatol. 2013; 68(3): e73-e82, doi: 10.1016/j.jaad.2011.08.021, indexed in Pubmed: 22000768.

31. Marionnet C, Pierrard C, Lejeune F, et al. Modulations of gene expression induced by daily ultraviolet light can be prevented by a broad spectrum sunscreen. J Photochem Photobiol B. 2012; 116: 37-47, doi: 10.1016/j.jphotobiol.2012.08.001, indexed in Pubmed: 22960577.

32. Mouret S, Leccia MT, Bourrain JL, et al. Individual photosensitivity of human skin and UVA-induced pyrimidine dimers in DNA. J Invest Dermatol. 2011; 131(7): 1539-1546, doi: 10.1038/jid.2011.47, indexed in Pubmed: 21430702.

33. Nechifor MT, Niculițe CM, Urs AO, et al. UVA irradiation of dysplastic keratinocytes: oxidative damage versus antioxidant defense. Int J Mol Sci. 2012; 13(12): 16718-16736, doi: 10.3390/ijms131216718, indexed in Pubmed: 23222638.
34. Ou-Yang H, Stamatas G, Kollias N. Dermal contributions to UVA-induced oxidative stress in skin. Photodermatol Photoimmunol Photomed. 2009; 25(2): 65-70, doi: 10.1111/j.1600-0781.2009.00403.x, indexed in Pubmed: 19292780.

35. Pattison DI, Rahmanto AS, Davies MJ. Photo-oxidation of proteins. Photochem Photobiol Sci. 2012; 11(1): 38-53, doi: 10.1039/c1 pp05164d, indexed in Pubmed: 21858349 .

36. Pittayapruek $P$, Meephansan J, Prapapan $O$, et al. Role of matrix metalloproteinases in photoaging and photocarcinogenesis. Int J Mol Sci. 2016; 17(6), doi: 10.3390/ ijms17060868, indexed in Pubmed: 27271600.

37. Prasedya ES, Syafitri SM, Geraldine BA, et al. UVA photoprotective activity of brown macroalgae. Biomedicines. 2019; 7(4): 77, doi: 10.3390/biomedicines7040077, indexed in Pubmed: 31569807.

38. Quan T, Little E, Quan H, et al. Elevated matrix metalloproteinases and collagen fragmentation in photodamaged human skin: impact of altered extracellular matrix microenvironment on dermal fibroblast function. J Invest Dermatol. 2013; 133(5): 1362-1366, doi: 10.1038/ jid.2012.509, indexed in Pubmed: 23466932.

39. Rünger TM, Farahvash B, Hatvani Z, et al. Comparison of DNA damage responses following equimutagenic doses of UVA and UVB: a less effective cell cycle arrest with UVA may render UVA-induced pyrimidine dimers more mutagenic than UVB-induced ones. Photochem Photobiol Sci. 2012; 11(1): 207-215, doi: 10.1039/c1 pp05232b, indexed in Pubmed: 22005748.

40. Schuch AP, Lago JC, Yagura T, et al. DNA dosimetry assessment for sunscreen genotoxic photoprotection. PLoS One. 2012; 7(6): e40344, doi: 10.1371/journal.pone.0040344, indexed in Pubmed: 22768281.

41. Scott TL, Christian PA, Kesler MV, et al. Pigment-independent cAMP-mediated epidermal thickening protects against cutaneous UV injury by keratinocyte proliferation. Exp Dermatol. 2012; 21(10): 771-777, doi: 10.1111/exd.12012, indexed in Pubmed: 23078399.

42. Wurm EMT, Longo C, Curchin C, et al. In vivo assessment of chronological ageing and photoageing in forearm skin using reflectance confocal microscopy. Br J Dermatol. 2012; 167(2): 270-279, doi: 10.1111/j.1365-2133.2012.10943.x, indexed in Pubmed: 22428802.

43. Xia Q, Chiang HM, Yin JJ, et al. UVA photoirradiation of benzo[a]pyrene metabolites: induction of cytotoxicity, reactive oxygen species, and lipid peroxidation. Toxicol Ind Health. 2015; 31(10): 898-910, doi: 10.1177/0748233713484648, indexed in Pubmed: 23552265. 